On the other hand, the animals weaned at 3 weeks were the heaviest at 9 weeks $(20 \mathrm{~kg}$ versus I $8.8 \mathrm{~kg}$ ). The choc due to weaning was then largely compensated, but this was not the case for the animals weaned at 5 weeks. Total feed intake was higher in the animals weaned at $2 \mathrm{I}$ days $(235 \mathrm{~kg}$ versus $198 \mathrm{~kg}$ feed/litter). Correlatively, the feed intake of the sows was lower for the same treatment (9I $\mathrm{kg}$ in the case of weaning at 3 weeks versus $\mathrm{I} 66 \mathrm{~kg}$ at 5 weeks). The age at weaning had no effect on the fertility of the sows.

From an economic point of view, feeding costs were slightly higher in the case of weaning at 3 weeks but this was compensated by a higher growth rate. Consequently, weaning at $2 \mathrm{I}$ days allows production of a greater number of piglets per sow and per year than weaning at 35 days (about T.5 piglets) without raising the feeding costs.

\title{
Étude de l'efficacité pRatique en élevage D'UN NEUROLEPTIQUE SUIVANT L'INTENSITÉ DE L'AGRESSION SUbie PAR LES ANimaUX AU MOMENT de L'ENTRÉ EN PORCherie
}

\author{
R. DANTZER \\ Station de Pharmacologie-Toxicologie, I. N. R. A., \\ Saint-Martin du Touch 180, Chemin de Tournefeuille,
} 31300 Toulouse

\section{RÉSUMÉ}

Afin d'apprécier l'intérêt éventuel de l'utilisation des neuroleptiques en élevage dans la prévention des troubles consécutifs aux agressions résultant du changement de porcherie après le sevrage, un essai clinique double aveugle a été réalisé, avec deux types d'agressions : un transport de faible durée ou un transport de longue durée intervenant après le regroupement des animaux et avant l'entrée en porcherie de préengraissement. Pour l'analyse des résultats, un schéma séquentiel a été retenu, l'unité expérimentale étant représentée par le lot et les critères de jugement par l'évolution des performances zootechniques (gain quotidien moyen et indice de consommation) au cours des 30 jours de la période de préengraissement.

En présence d'une agression de faible intensité, la Propericiazine (à la dose de $0,5 \mathrm{mg} / \mathrm{kg}$ en injection intramusculaire) entraîne une diminution des performances zootechniques par un effet défavorable sur la croissance, tandis qu'après une agression importante, les animaux traités se révèlent supérieurs aux animaux témoins au moins pendant les 17 premiers jours de l'entrée en porcherie.

Ces résultats montrent qu'avant d'utiliser une drogue en élevage, il est nécessaire de tester son efficacité pratique par un essai clinique utilisant une méthodologie appropriée.

\section{SUMMARY}

\section{PRACTICAL, EFFECTIVENESS OF A NEUROLEPTIC AGENT UPON WEANED PIGLETS}

To study the effectiveness of a neuroleptic agent in alleviating the problems following the mixing and moving of weaned piglets from one piggery to another, a double blind clinical trial has been used with two different procedures $i . e$. either a short or a long lasting transport both occuring after the pigs were mixed, but before they entered the new piggery. 
A sequential analysis has been performed on the data based upon the results from each group of pigs and the measurements made were mean growth rate and food conversion ratio during the prefattening period of 30 days.

Propericiazine $(0,5 \mathrm{mg} / \mathrm{kg}$ IM) was found to reduce growth rate when used after the short journey but increased growth rate and food conversion after the long journey when compared with controls injected with a placebo. The results indicate the usefulness of appropriately planned clinical trials.

\title{
MODIFICATIONS DANS LES RYTHMES D'ACTIVITÉ MOTRICE DES PORCELETS LORS DE L'ENTRÉE EN PORCHERIE
}

\author{
R. DANTZER \\ Station de Pharmacologie et de Toxicologie, I. N. R. A., \\ Saint-Martin du Touch, 180, Chemin de Tournefeuille, \\ 31300 Toulouse
}

\section{RÉSUMÉ}

La mesure de l'activité motrice de porcelets sevrés par un dispositif utilisant des cellules photoélectriques a permis de mettre en évidence l'existence d'un rythme nycthéméral caractérisé par 2 pics. le pic matinal était inférieur au pic vespéral. L'étude des variations de ce rythme au cours des 20 premiers jours de l'entrée en porcherie a été effectuée sur les paramètres suivants : l'importance de l'activité nocturne par rapport à l'activité quotidienne, le nombre total d'impulsions obtenu par jour; des indices de répartition de l'activité, calculés sur les percentiles 25 et 75 et la médiane, à partir de la courbe cumulative des pourcentages d'activité horaire. Les phénomènes d'adaptation se traduisent par une hyperactivité surtout manifeste pendant les trois premiers jours, une activité nocturne faible et un étalement de l'activité diurne dans le temps peu important. Le paramètre le plus sensible semble être représenté par l'indice relatif qui traduit la comparaison de la répartition de l'activité matinale à celle de l'activité vespérale et qui nécessite huit jours pour atteindre une valeur stable.

Ces résultats témoignent de l'importance et de l'intérêt d'une approche en termes de rythmes biologiques dans l'appréciation de la durée et de l'intensité des phénomènes d'adaptation chez le porc.

\section{SUMMARY}

\section{CHANGES IN LOCOMOTOR ACTIVITY OF WEANED PIGLETS AFTER MOVING IN A NEW PIGGERY}

Locomotor activity of weaned piglets has been studied using a photocell technique. A circadian rhythm was observed in which the main features were predominance of diurnal activity and existence of two peaks, $i . e$. a morning peak and a larger afternoon peak. Changes in 